\title{
Functional plasticity associated with language learning in adults
}

Kshipra Gurunandan $*^{\mathrm{a}}$, Manuel Carreiras ${ }^{\mathrm{a}, \mathrm{b}, \mathrm{c}}$ and Pedro M. Paz-Alonso ${ }^{*^{\mathrm{a}}}$

(a) BCBL, Basque Center on Cognition, Brain and Language, Donostia-San Sebastian, Spain

(b) IKERBASQUE, Basque Foundation for Science, Bilbao, Spain

(c) Department of Basque Language and Communication, EHU/UPV, Bilbao, Spain

*Correspondence should be addressed to:

Kshipra Gurunandan or Pedro M. Paz-Alonso, BCBL Basque Center on Cognition, Brain and Language, Paseo Mikeletegi 69, 20009 Donostia-San Sebastian, Gipuzkoa, Spain. Tel: +34 943-309-300, fax: +34 943-309-052, e-mails: k.gurunandan@bcbl.eu, p.pazalaonso@bcbl.eu

Declaration of interests: none. 


\begin{abstract}
Learning a new language in adulthood is increasingly common and among the most difficult tasks attempted by adults. Adult language learners thus offer an excellent window into the nature of learning-dependent plasticity. The present functional magnetic resonance imaging (fMRI) study was aimed at characterising functional neuroplasticity in adults at different stages of learning a second language (L2). To this end, a total of 34 adults, either intermediate or advanced L2 learners, underwent MRI scanning while performing a semantic judgement task with print and speech stimuli. Three separate analytical approaches were used to comprehensively map neural differences: print-speech convergence, L1-L2 similarity, and functional connectivity with language control regions. Results revealed that (i) print-speech convergence was not affected by L2 proficiency level, (ii) L1-L2 similarity was significantly higher in intermediate than in advanced L2 learners, and (iii) functional coupling of language and language control areas was higher in the advanced relative to the intermediate group during reading comprehension. The results point to significant functional differences between intermediate and advanced language learners, indicating that, even well into adulthood, increasing L2 proficiency modulates the functional similarity between L1 and L2 and the connectivity between language comprehension and language control regions, particularly in reading comprehension.
\end{abstract}

Keywords: adult plasticity, functional MRI, language proficiency 


\section{Introduction}

Learning a new language in adulthood is becoming increasingly common and is typically a complex and effortful process. Adult language learners thus offer an excellent window into a range of learning-dependent neural changes occurring in an ecological context. Language learning in adults has often been studied by using artificial languages or discrimination tasks focusing on specific skills such as word learning (López-Barroso et al. 2013; Plante et al. 2015; Li et al. 2018), foreign speech sound discrimination (Golestani and Zatorre 2004, 2009), and learning of pitch patterns (Wang et al. 2003; Wong et al. 2007). These experimental approaches have provided valuable insights into the neural changes concomitant with these aspects of L2 learning, but the multi-dimensionality of language also means that investigating the full neural impact of real-world language learning requires ecologically valid experiments.

Classical studies have found that the age of acquisition and proficiency in the second language (L2) modulate functional and structural neural differences in bilingual adults (e.g. Kim et al. 1997; Mechelli et al. 2004). The influence of these two factors has been well studied in adults who acquired their L2 as children (e.g. Perani et al. 1998, 2003, 2005; Chee et al. 2001; Wartenburger et al. 2003; Liu and Cao 2016), but fewer studies have examined adults who are actively learning a new language. Learning-dependent neuroplasticity has been seen in adults within the first 3-5 months of learning a new language (Stein et al. 2009, 2012; Martensson et al. 2012; Schlegel et al. 2012; Xiang et al. 2015; Chai et al. 2016; Barbeau et al. 2017), but less attention has been paid to what happens next. After the initial effort of L2 learning, do language networks continue to change in adults progressing from the intermediate to the advanced stages of L2 learning?

To examine learning-dependent plasticity in adults past the initial stage of L2 learning, we studied two groups of adults enrolled in the same language school: one group 
from intermediate level classes and the other from advanced level classes. The study sample was controlled for both extra-linguistic and linguistic factors - all participants were native to the region and were learning a local language that differs substantially from their native language in morphology and syntax, but has largely overlapping phonology and orthography. We used a semantic judgement task with single words presented visually and auditorily to map the reading and speech comprehension networks in the participants' native language (L1) and in the language being learnt (L2). To comprehensively map changes in the neural representations of L1 and L2, we examined three things: (i) functional convergence of reading and speech comprehension, (ii) functional similarity of L1 and L2, and (iii) functional connectivity between classical language regions and language control regions.

First, we examined the role of language proficiency in the functional convergence of print- and speech-comprehension. It has been shown that print-speech convergence varies with reading skill in monolingual children and adults — above and beyond general activation for printed and spoken stimuli — since skilled reading involves integration of printprocessing with pre-existing spoken language networks (Shankweiler et al. 2008; Frost et al. 2009; Preston et al 2016). Not limited to specific languages, this convergence between reading and speech comprehension networks has been found in adult native speakers of highly contrasting languages with different writing systems, levels of orthographic depth, and morphological properties, and is thus considered a universal signature of proficient reading (Rueckl et al. 2015). A study with late bilingual-biliterates (bilinguals whose languages use different writing systems, e.g., English and Chinese; see Singh et al. 2016) extended these results to L2, finding differences in the pattern of print-speech convergence between L1 and L2 that were indicative of the greater effort and lower automaticity of L2 reading in a new writing system (Brice et al. 2019). However, monolinguals and many bilingual-biliterates learn to speak before they learn to read, which is not the case for late bilinguals whose L2 
uses the same writing system as their L1. Hence, the effect of overall L2 proficiency on convergence of reading and spoken language networks when reading is already proficient remains an important open question for the reliability of print-speech convergence in L2. In the current study, all participants were proficient readers in their L1, and their L2 uses the same writing system and has phonology and a transparent orthography largely overlapping with the L1, thus allowing us to specifically examine the effect of language proficiency on print-speech convergence, independent of reading skill. We expected to find substantial printspeech convergence reflecting proficient reading in both L1 and L2, and hypothesised that any effects specifically due to increased L2 proficiency would emerge as differential patterns of convergence in the intermediate and advanced groups.

Second, we examined the effect of L2 proficiency on the similarity of L1 and L2 activation patterns. While L1 and L2 have been found to utilise common semantic "hubs" in proficient bilinguals (Chee et al. 1999; Perani and Abutalebi 2005; Buchweitz et al. 2012; Correia et al. 2014), psycholinguistic studies make the case for L1-mediated access to L2 in the early stages of L2 learning, with L1-dependence decreasing in the later stages (Kroll and Stewart 1994; Kroll et al. 2010). This could suggest higher similarity between L1 and L2 activation in the intermediate L2-learners due to L1-dependence of L2, and greater separation in the advanced learners as the L2 became less dependent on L1. Previous studies of bilinguals have found substantial proficiency-dependent variability in L2 activation, particularly in lateralisation (Dehaene et al. 1997; Abutalebi et al. 2001; Hull and Vaid 2007). To take this variability into account and to examine the idea that similarity of L1 and L2 vary as a function of L2 proficiency, we used laterality indices to compare L1 and L2 activation in the language network areas presented in prominent neuroanatomical models of language (e.g., Lau et al. 2008; Hagoort 2013). By restricting the analysis to classical language regions, we aimed to avoid conflating activation in regions associated with language control 
(addressed separately in the subsequent analysis). Further, we analysed the contributions of the dorsal and ventral pathways to laterality changes in the reading and speech comprehension networks. The dorsal and ventral streams are known to subserve phonological processing and lexico-semantic mapping, respectively (Jobard et al. 2003; Hickok and Poeppel 2004; Schlaggar and McCandliss 2007; Friederici 2012; Oliver et al. 2017), and may thus be differentially modulated in the course of language learning.

Finally, we examined the role of L2 proficiency and exposure in functional coupling of the language regions and language control regions. One of the recurring themes in bilingual language processing is the recruitment of areas not typically included in the classical language networks, such as the dorsolateral prefrontal cortex (dlPFC) and anterior cingulate cortex (ACC), which are thought to be involved in control processes associated with language (e.g. Chee et al. 2001; Abutalebi et al. 2007; Marian et al. 2007; Hernandez 2009). This effect, at least in the lexico-semantic domain, appears to be independent of the age of L2 acquisition and to rely primarily on L2 proficiency and exposure (see Abutalebi et al. 2001; Indefrey 2006 for reviews). Supporting the role of L2 exposure, a verbal production study found more extensive activation in left and right prefrontal areas associated with lower L2 exposure in early, highly proficient bilinguals (Perani et al. 2003). If more diffuse activation of control areas is associated with lower L2 proficiency and exposure, this might imply that with increasing proficiency and exposure, functional connectivity between control areas and classical language regions in L2 learners becomes stronger. To examine functional interactions between the dIPFC and ACC and the classical language areas during L2 processing, we conducted functional connectivity analyses. We expected to observe stronger functional coupling in advanced L2-learners who had more experience in the L2 than the intermediate group. 


\section{METHODS}

\subsection{Participants}

The final study sample consisted of 29 right-handed native Spanish speakers (mean age $=43.7 \pm 9.7$ years; 15 female) studying Basque in the same language school. Data from five other participants was excluded due to excessive head motion during imaging. Participants were native to the Basque Country, Spain, and had grown up primarily exposed to Spanish (L1) at home and in school. They were now living in Spanish-Basque bilingual environments, and enrolled in Basque (L2) classes at either the Common European Framework of Reference for Languages (CEFR) A2 level (intermediate group, $n=14$ ) or C1 level (advanced group, $\mathrm{n}=15$ ), and had uniformly high performance in class. Language proficiency was further assessed using objective and subjective measures. Participants performed a picture-naming task in their two languages - an adaptation of the Boston Naming Test (Kaplan et al. 1983) controlled for cognates across L1 and L2 — and completed a language background questionnaire in which they rated their proficiency and percentage of daily exposure outside the classroom to each language (Table 1).

The two groups were matched on age, gender, IQ and Spanish proficiency (Table 1). Participants had limited knowledge of English or other languages, with little day-to-day exposure to them, and there was no difference between groups in this regard $(p=0.83)$. All participants had normal or corrected-to-normal vision, and no history of neurological or psychiatric disorders. In compliance with the ethical regulations established by the BCBL Ethics Committee and the guidelines of the Helsinki Declaration, all participants gave written informed consent prior to taking part in the experiment, and received monetary compensation for their participation. 
Table 1. Participant demographics and linguistic scores by group

\begin{tabular}{|c|c|c|c|}
\hline & Intermediate L2 group & Advanced L2 group & $p$-value \\
\hline Age & $42.86(10.10)$ & $44.53(10.51)$ & 0.66 \\
\hline Gender & 7 female, 7 male & 8 female, 7 male & 0.86 \\
\hline IQ & $117(13.78)$ & $122(9.43)$ & 0.12 \\
\hline L1 proficiency & $99.35(1.88)$ & $99.64(0.77)$ & 0.61 \\
\hline L2 proficiency & $52.6(14.66)$ & $87.96(10.58)$ & 0.00 \\
\hline L1 exposure & $85.23(16.96)$ & $71.31(24.43)$ & 0.11 \\
\hline L2 exposure & $8.54(8.32)$ & $23.46(22.93)$ & 0.04 \\
\hline
\end{tabular}

1. Values correspond to the mean with standard deviation in parentheses.

2. $p$-values correspond to the t-test between groups (chi-square test for gender).

3. L1+L2 exposure does not sum up to $100 \%$ since a few participants reported minor exposure to foreign languages English/French/German (difference between groups $p=0.83$ )

\section{2 fMRI Task}

Inside the MRI scanner, participants performed a semantic animacy judgement task (living/non-living) in their L1 and L2, indicating their responses via button presses using their dominant (right) hand. To avoid language-switching effects, the languages were separated and their order was counterbalanced across participants. We used an event-related fMRI design, with three runs per language. Each run had 48 stimuli with inter-mixed reading and listening trials. Printed stimuli subtended visual angles of $4^{\circ}-6^{\circ}$ and were all displayed for $1000 \mathrm{~ms}$, while auditory stimuli had a mean duration of $565 \mathrm{~ms}$ (sd=86 ms). Stimuli were high frequency, concrete, imageable nouns with an even split between living and non-living items. The baseline condition consisted of a fixation cross presented in the centre of the screen. 


\subsection{MRI Data Acquisition}

Whole-brain MRI data was acquired using a 3-T Siemens Magnetom Trio whole-body MRI scanner and a 32-channel head coil at the Basque Center on Cognition, Brain and Language (BCBL). Padded headphones were used to dampen background scanner noise and enable clear transmission of the auditory stimuli. Participants viewed the print stimuli on a screen via a mirror mounted on the head coil. To limit head movement, the head coil was padded with foam and participants were asked to remain as still as possible.

Functional MRI was acquired in the course of six separate runs using a gradient-echo echo-planar pulse sequence with the following parameters: TR $2000 \mathrm{~ms}$, TE $30 \mathrm{~ms}, 32$ axial slices with a $3.4 \times 3.4 \times 4 \mathrm{~mm}$ voxel resolution, $10 \%$ inter-slice gap, flip angle (FA) $=80^{\circ}$, field of view $(\mathrm{FoV})=220 \times 220 \mathrm{~mm}, 64 \times 64$ matrix. 186 volumes were collected for each of the six functional runs. Prior to each scan, four volumes were discarded to allow for T1equilibration effects. To improve estimation of the resting baseline in functional analyses, functional runs contained three silent fixation periods of $20 \mathrm{~s}$ each. Within each functional run, the order of the trials (reading and listening conditions) and the inter-trial intervals of variable duration (4-20 s) corresponding to the baseline MR frames (30\% of total collected functional volumes) were determined by an algorithm designed to maximise the efficiency of the recovery of the blood oxygen level dependent response (optseq2, Dale 1999). Structural T1-weighted images were acquired with a MPRAGE sequence with TR $=2530 \mathrm{~ms}$, TE $=$ $2.97 \mathrm{~ms}$, inversion time $=1100 \mathrm{~ms}, \mathrm{FA}=7^{\circ}, \mathrm{FoV}=256 \times 256 \mathrm{~mm}, 176$ slices and voxel size $=1 \mathrm{~mm}^{3}$.

\subsection{MRI Data Analysis}

Standard SPM8 (Wellcome Department of Imaging Neuroscience, London) preprocessing routines and analysis methods were employed. Images were first corrected for 
differences in timing of slice acquisition and then realigned to the first volume using rigidbody registration. Each subject's functional volumes were spatially smoothed with a 4-mm full-width half-maximum (FWHM) Gaussian kernel. Next, motion parameters obtained from realignment were used to inform a volume repair procedure (ArtRepair; Stanford Psychiatric Neuroimaging Laboratory) that identified bad volumes on the basis of scan-to-scan movement $(>1 \mathrm{~mm})$ and signal fluctuations in global intensity $(>1.3 \%)$, and corrected bad volumes via interpolation between the nearest non-repaired scans. Data from five subjects requiring more than $20 \%$ of their volumes to be repaired were discarded. The number of corrected volumes was similar between groups $(p=0.19)$. After volume repair, high-resolution anatomical T1 images and functional volumes were coregistered and spatially normalised to T1 and echo-planar imaging templates, respectively, to enable anatomical localisation of the activations. Templates were based on the MNI305 stereotaxic space (Cocosco et al. 1997), an approximation of Talairach space (Talairach and Tournoux 1988). The normalisation algorithm used a 12-parameter affine transformation together with a nonlinear transformation involving cosine basis functions. During normalisation, the volumes were sampled to 3-mm cubic voxels. The resulting volumes were then spatially smoothed with a 7-mm FWHM Gaussian kernel. Finally, time series were temporally filtered to eliminate contamination from slow frequency drift (high-pass filter with cut-off period of $128 \mathrm{~s}$ ).

Statistical analyses were performed on individual subject data using the general linear model (GLM). fMRI time series data were modelled by a series of impulses convolved with a canonical haemodynamic response function. Six motion parameters for translation $(\mathrm{x}, \mathrm{y}, \mathrm{z})$ and rotation (yaw, pitch, roll) were included as covariates of non-interest in the GLM. Each trial was modelled as an event, time-locked to the onset of the presentation of each stimulus. Error responses were modelled separately. The remaining functions were used as covariates in the GLM, along with a basic set of cosine functions that high-pass filtered the data, and a 
covariate for session effects. The least-squares parameter estimates of the height of the bestfitting canonical HRF for each study condition were used in pairwise contrasts. Contrast images from each subject were submitted to group analyses. At the group level, whole-brain contrasts between conditions were computed by performing one-sample t-tests on the images, treating subjects as a random effect. Brain coordinates throughout the text, as well as in tables and figures, are reported in MNI atlas space (Cocosco et al. 1997).

\subsubsection{Print-speech convergence}

We first obtained each subject's whole-brain contrasts Print_correct>Rest and Speech_correct $>$ Rest in each language condition, with a voxel-wise corrected false discovery rate (FDR) threshold set at $q<0.05$, and used these contrasts to compute voxel-to-voxel Pearson correlations (WFU Biological Parametric Mapping Toolbox, Casanova et al. 2007) across subjects within each proficiency group. We used two methods to test for differences between the intermediate and the advanced groups in each language: (i) we performed minimum conjunction in each subject to retain only voxels that were significantly active in both the reading and speech conditions, and carried out statistical non-parametric mapping (SnPM13 toolbox, Nichols and Holmes 2001) on the resulting images to identify voxels that differed significantly between groups or languages, and (ii) calculated a correlation coefficient (Pearson's $r$ ) between voxels in print and speech conditions for each subject and used a 2x2 ANOVA to test for group differences in either language.

\subsubsection{L1-L2 similarity}

To test similarity of $\mathrm{L} 1$ and $\mathrm{L} 2$ activation in the language network regions, we correlated the laterality indices of activation in each language. Laterality is calculated by dividing the difference between activation in each hemisphere by the sum, resulting in an 
index between -1 (fully right-lateralised activation) and +1 (fully left-lateralised activation). In line with the latest recommendations (Bradshaw et al. 2017), we used a thresholdindependent method to calculate the index (LI-Toolbox, Wilke and Lidzba 2007). We chose six bilateral anatomical regions of interest (ROI) from classical neuroanatomical language models (e.g., Lau et al. 2008; Hagoort 2013): inferior frontal gyrus (IFG) pars orbitalis, IFG pars triangularis, IFG pars opercularis, inferior parietal lobule (IPL), superior temporal gyrus (STG), and ventral occipito-temporal cortex (vOTC), and masked each subject's whole-brain contrasts for the reading and speech conditions with the selected anatomical regions from the AAL atlas (Tzourio-Mazoyer et al. 2002). We acquired laterality indices for three networks: (i) the language network consisting of all six ROIs, (ii) the dorsal phonological network consisting of the IPL, STG and IFG pars opercularis, and (iii) the ventral lexical network consisting of the IFG pars orbitalis, IFG pars triangularis, and vOTC. We then calculated correlation between indices (using Pearson's $r$ ) to test the similarity between L1 and L2 language networks within each group, as well as (i) a twosample t-test to test between-group differences in L1-L2 laterality, and (ii) Cohen's $d$ to test for group differences in each language.

\subsubsection{Functional Connectivity}

We assessed functional connectivity using the beta-series correlation method (Rissman et al. 2004) implemented in SPM8 with custom MATLAB scripts. The canonical HRF in SPM was fitted to each occurrence of each condition and the resulting parameter estimates (beta values) were sorted according to the study conditions to produce a conditionspecific beta series for each voxel. Pairwise connectivity was calculated between selected ROIs in the bilateral language networks and control regions for each participant and condition. Since the correlation coefficient ranges from -1 to +1 , an arc-hyperbolic tangent 
transform (Fisher 1921) was applied to these beta-series correlation values to make its null hypothesis sampling distribution approach that of the normal distribution. To test for group differences in functional connectivity strength as a function of our experimental design, the normally distributed Fisher's Z values were submitted to group comparisons within each of the conditions of interest: L1 reading, L1 speech, L2 reading, and L2 speech. The regions selected for these functional connectivity analyses included the previously described bilateral language network regions (IFG pars orbitalis, triangularis, opercularis, STG, IPL, and vOTC) and two bilateral cognitive control regions: dorsolateral prefrontal cortex (dlPFC) and anterior cingulate cortex (ACC). Functionally defined ROIs were identified from the wholebrain contrast All_Correct_Trials $>$ Rest with a voxel-wise FDR-corrected threshold $\mathrm{q}<0.05$, and 4-mm radius spheres were centred at the highest local maxima within each ROI, to ensure that differences in the functional connectivity between regions were not affected by the size of the ROIs. This gave us five spheres for cognitive control: two in the left dlPFC and one in the right, and one each in the left and right ACC.

\section{RESULTS}

\subsection{Behavioural In-scanner Performance}

Mixed-model ANOVAs were separately conducted on the behavioural measures of the fMRI task, i.e. accuracy (percentage of correct responses) and reaction times, with Group (intermediate, advanced) as a between-subjects factor and with Language (L1, L2) and Modality (print, speech) as within-subjects factors. The first ANOVA for accuracy revealed a Group $x$ Language interaction in the accuracy of participants' responses during the fMRI task $(\mathrm{F}(1,23)=20.65, p=0.0001)$. Post-hoc simple-effect analyses showed that the intermediate and advanced L2-learners exhibited no difference in accuracy in their L1 $(\mathrm{t}(18.33)=-1.44$, $p=0.17)$, but a significant difference in $\mathrm{L} 2(\mathrm{t}(21.94)=5.02, p=0.00005)$, with the intermediate 
group showing significantly lower L2 accuracy than the advanced group (Figure 1a). This effect was observed in both modalities, reading and speech. The ANOVA for participants' reaction times found only a main effect of Language (Figure 1b), with both groups significantly slower in their L2 compared to their L1 $(\mathrm{F}(1,23)=44.5, p=0.0000008)$.
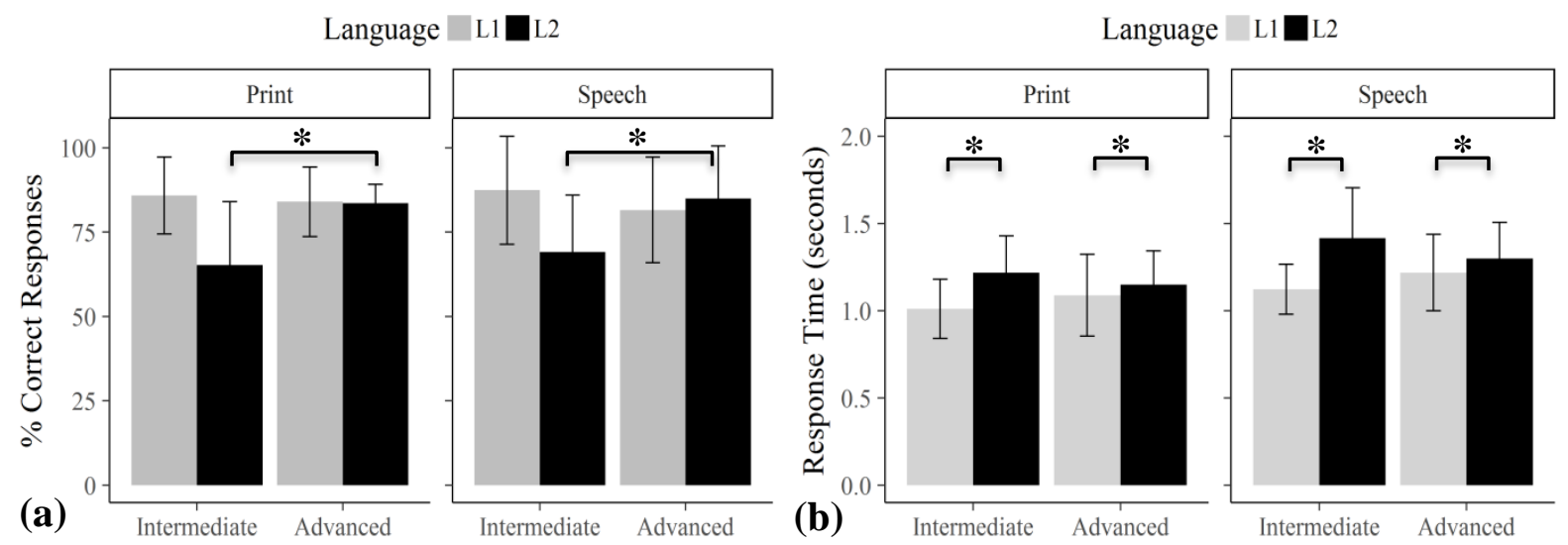

Figure 1. Behavioural results of the semantic judgement task inside the scanner revealed (a) Group x Language interaction in accuracy of responses, and (b) main effect of Language in reaction times of participants. Error bars represent standard deviation and asterisks statistically significant differences at $p<0.05$

\subsection{Print-speech Convergence}

Print-speech convergence in each language was calculated from the subjects' wholebrain contrasts Print_correct>Rest and Speech_correct>Rest using voxel-to-voxel Pearson correlation. The bilateral striate and extrastriate regions were significantly active only for the reading conditions, while posterior parts of the superior temporal gyrus (STG), including primary auditory cortex, were active only for the listening conditions. In L1 (Figure 2a), convergence of printed and spoken language processing $(r>0.31, p<0.05)$ was found in bilateral areas associated with both phonological and semantic processing such as the IFG, middle temporal gyrus (MTG) and STG, and IPL. In L2 (Figure 2b), the pattern of convergence encompassed similar areas, but with slightly more extensive visual cortex activation for the reading condition, and greater convergence in the dlPFC in comparison to L1, which showed greater convergence in parietal regions. Neither the statistical non- 
parametric mapping nor the ANOVA revealed significant differences at $p<0.05$ threshold (FDR-corrected for SnPM) between the two groups in either language.
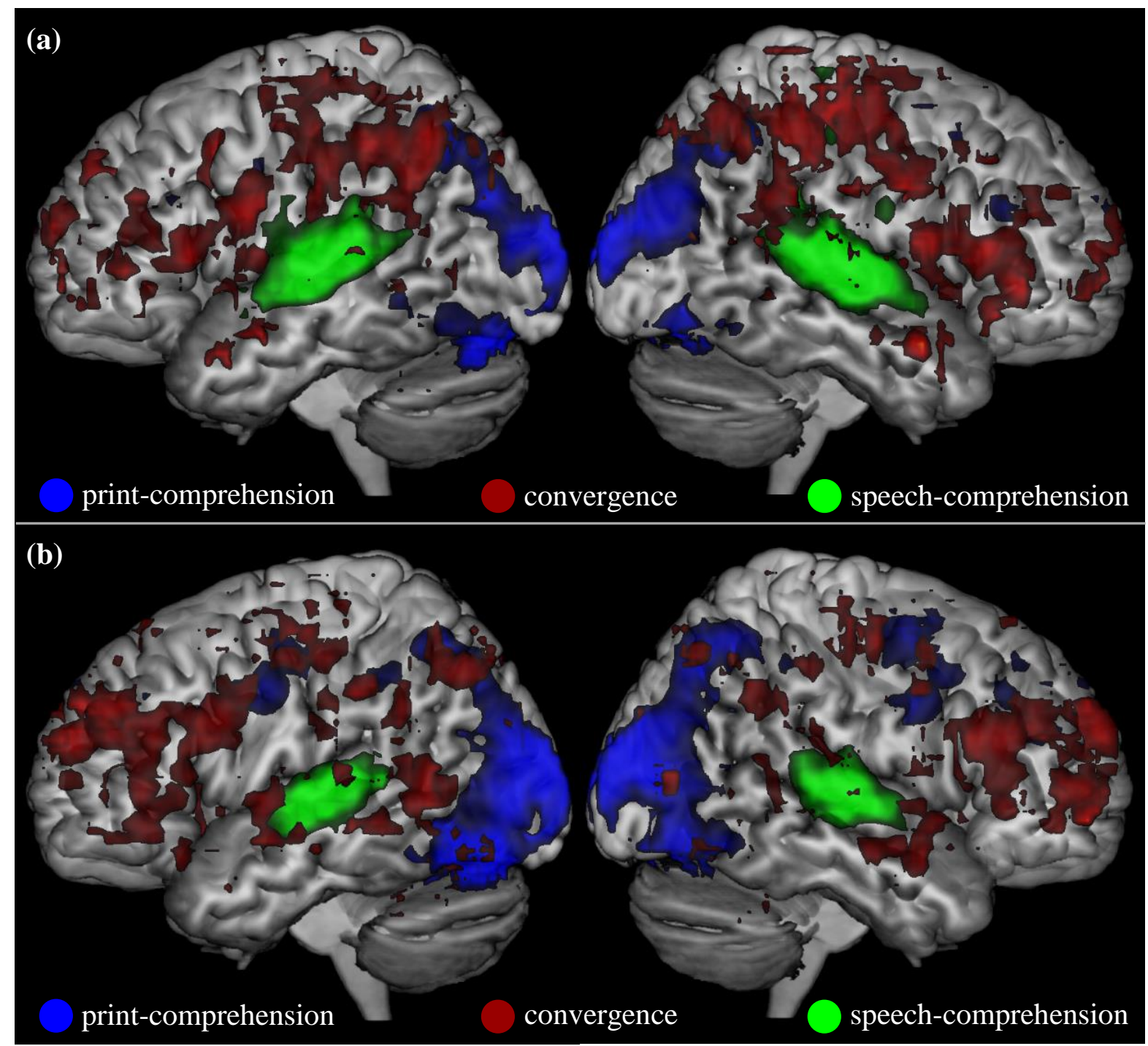

Figure 2. Print-speech convergence in (a) L1 and (b) L2

All subjects (n=29); FDR-corrected $q<0.05$; Pearson's $r>0.31, p<0.05$

\subsection{L1-L2 Similarity}

To investigate whether the intermediate and advanced groups displayed similar activation patterns in L1 and L2, we correlated the laterality indices for L1 and L2 activation in the language network regions within each group (Figure 3). Laterality indices of activation in L1 and L2 in each modality were calculated for each subject, giving us values between -1 
(completely right-lateralised activation) and +1 (completely left-lateralised activation). In the intermediate group, we found a positive correlation between L1 and L2 laterality in reading $(r=0.54, p=0.029)$ and speech comprehension $(r=0.72, p=0.001)$. In contrast, the advanced group exhibited a negative correlation in reading $(r=-0.46, p=0.048)$ and a non-significant correlation in speech comprehension $(r=0.43, p=0.951)$. A two-sample t-test of L1-L2 similarity confirmed a statistically significant difference between the groups in the language network in reading $(\mathrm{t}(19.51)=2.25, p=0.018)$ but not in speech comprehension $(\mathrm{t}(25.98)=1.79, p=0.15)$.

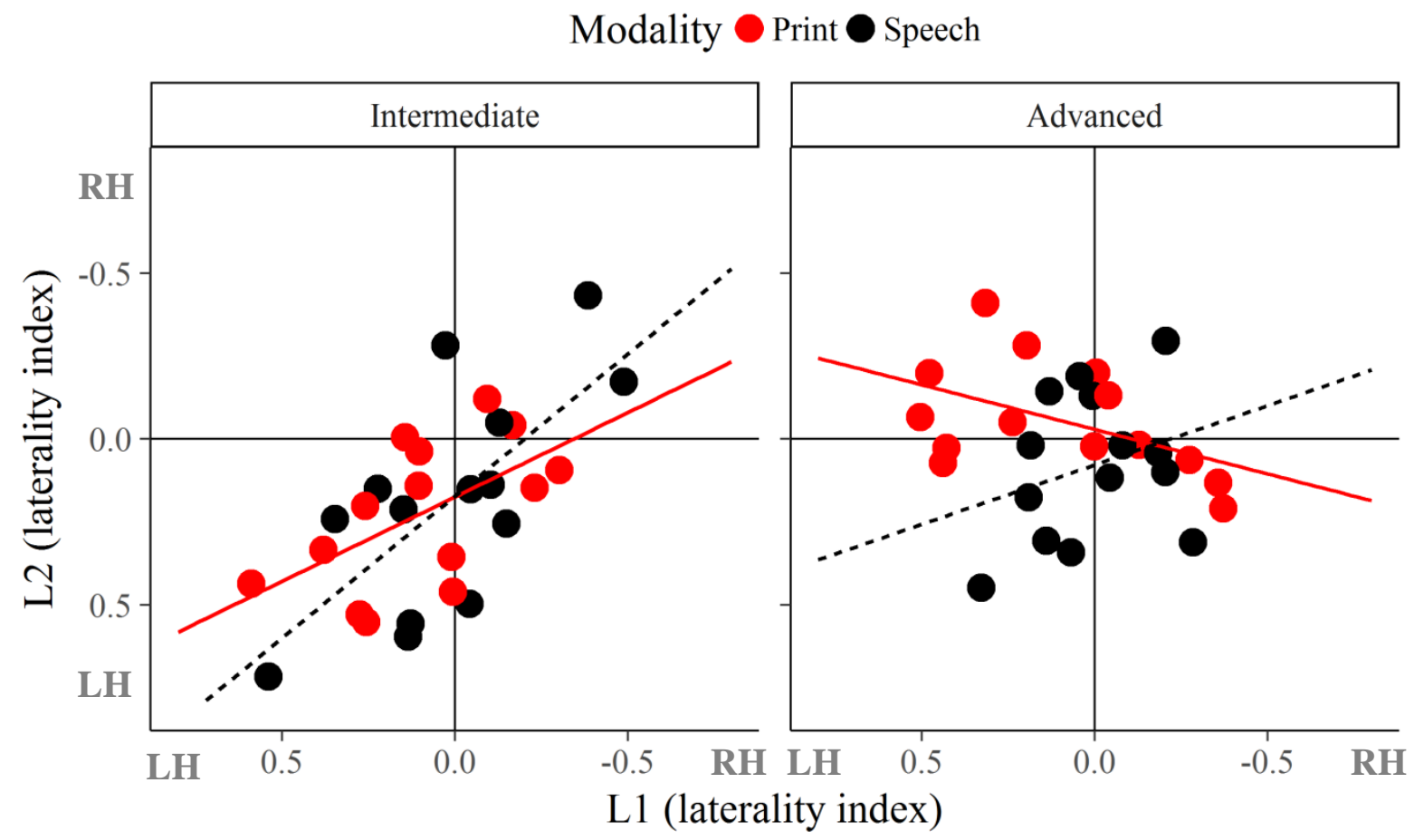

Figure 3. L1-L2 correlation of laterality indices. LH indicates greater activation in the left hemisphere; $\mathrm{RH}$ indicates greater activation in the right hemisphere.

To further verify that the difference in activation patterns between groups came from differences in L2 and not differences in L1, we calculated the effect sizes (Cohen's $d$ ) of the indices. We found negligible differences in L1 lateralisation between the intermediate and advanced L2-learner groups, and large and medium effects in L2 lateralisation (Table 2), with the advanced group showing more bilateral activation in L2 than the intermediate group. In 
L2 reading, the dorsal and ventral networks both showed large effects of L2 proficiency, but in L2 speech comprehension, only the ventral network exhibited a medium effect of L2 proficiency.

Table 2. Cohen's $d$ : effect sizes of between-group differences in laterality

\begin{tabular}{|c|c|c|c|c|c|}
\hline \multirow{2}{*}{ Stimuli } & Network & \multicolumn{2}{|c|}{$\begin{array}{r}\text { Between-group difference in } \\
\text { L1 }\end{array}$} & \multicolumn{2}{|c|}{$\begin{array}{c}\text { Between-group difference in } \\
\text { large }\end{array}$} \\
\hline \multirow{4}{*}{ Print } & Language & negligible & $\mathbf{0 . 0 7}$ & $\mathbf{1 . 4 1}$ \\
\cline { 2 - 6 } & dorsal & negligible & 0.02 & large & 1.10 \\
\cline { 2 - 6 } & ventral & negligible & 0.06 & large & 0.98 \\
\hline \multirow{3}{*}{ Speech } & Language & negligible & $\mathbf{0 . 0 3}$ & small & $\mathbf{0 . 3 8}$ \\
\cline { 2 - 6 } & dorsal & negligible & 0.04 & small & 0.27 \\
\cline { 2 - 6 } & ventral & small & 0.27 & medium & 0.68 \\
\hline
\end{tabular}

\subsection{Functional Connectivity}

To examine functional interactions between the language network and languagecontrol regions, we performed pairwise connectivity analyses using the beta-series correlation method. In L2 reading, we found significantly ( $q<0.05$, FDR-corrected) stronger left dlPFC left STG, and left dIPFC - right IFG pars opercularis connectivity in the advanced L2learner group, compared to the intermediate L2-learner group (Figure 4). No group differences in coupling strength between regions were observed in L2 speech comprehension. 
Axial View

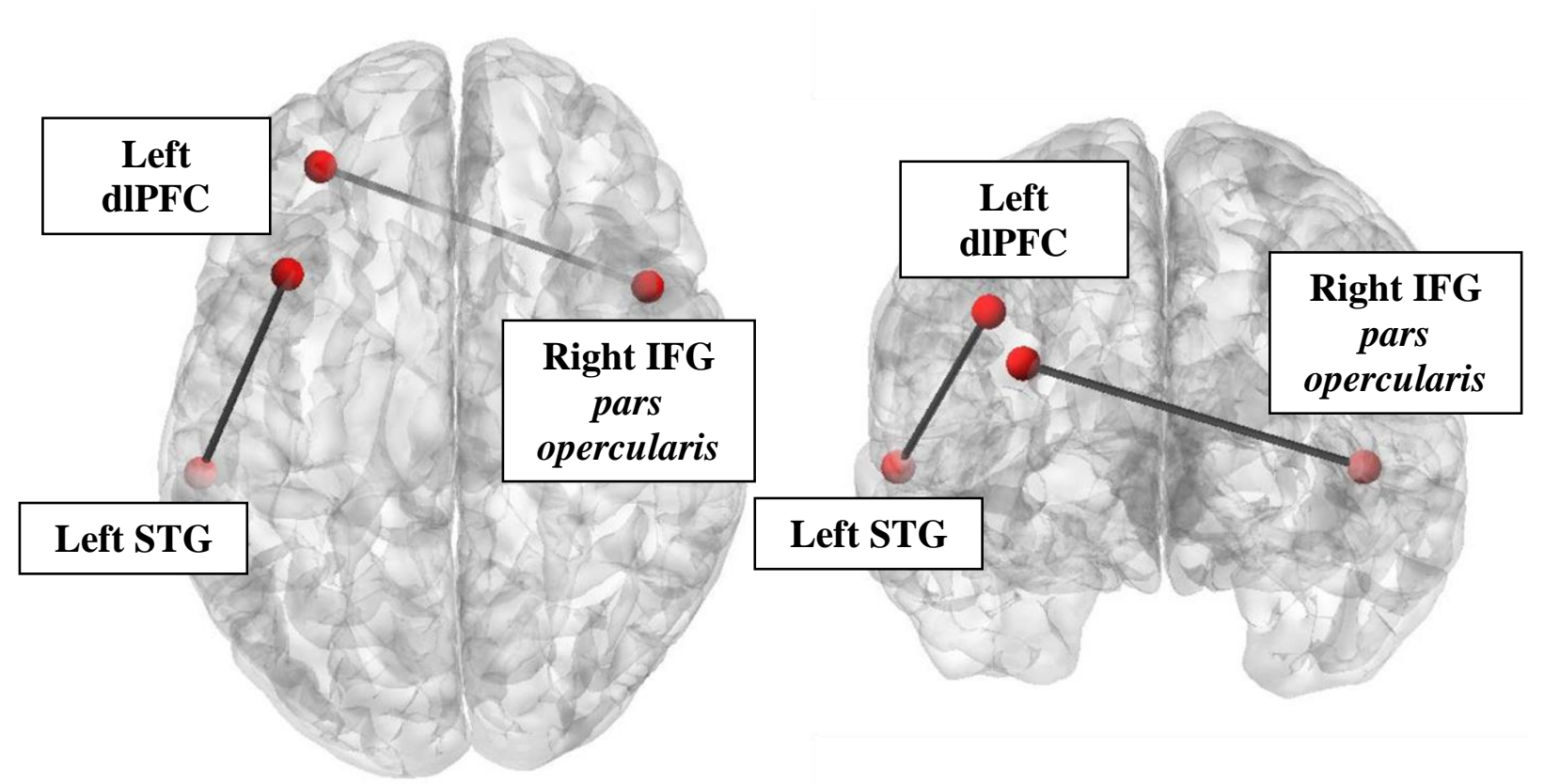

Figure 4. Advanced $>$ Intermediate functional connectivity in L2 reading ( $q<0.05$, FDR-corrected) $\mathrm{d} \mathrm{lPFC}=$ dorsolateral Prefrontal Cortex, IFG $=$ Inferior Frontal Gyrus, STG $=$ Superior Temporal Gyrus

\section{DISCUSSION}

In the current study, we examined functional differences between adult intermediate and advanced language learners. While structural and functional changes have previously been observed in young adults learning completely new languages, functional correlates of neural changes in higher proficiency adult language learners had yet to be investigated. To ensure sufficient proficiency differences, we used a cross-sectional design and studied two groups of adult language learners from intermediate and advanced level classes at the same language school. All participants were studying a local language with very different morphology and syntax, but similar phonology and the same writing system and a transparent orthography as in their native language, thus controlling for extra-linguistic factors such as immigration or foreignness, as well as the more sensory (visual and phonological) differences between languages. To ensure semantic access inside the scanner, we used a semantic judgement task with single words to separately map activation for each language. Our 
analytical approach included three measures: print-speech convergence, L1-L2 similarity, and functional connectivity with language control regions. We found that (i) print-speech convergence was not affected by L2 proficiency, (ii) L1-L2 similarity was significantly higher in intermediate than in advanced L2 learners, and (iii) functional coupling of language and language control areas was higher in the advanced relative to the intermediate group during reading comprehension. Collectively, our results point to significant functional differences between adult language learners in the intermediate and the advanced stages of learning, indicating that increasing L2 proficiency engenders plasticity well into adulthood.

\subsection{Print-speech convergence is unaffected by $L 2$ proficiency}

In both L1 and L2, we found significant convergence of reading and speech comprehension in classical language areas. In L1, the convergence was consistent with previous findings in native speakers of different languages (Rueckl et al. 2015), indicating that print-speech convergence in L1 was not affected by L2 acquisition. In L2, we saw a convergence pattern very similar to L1, with more extensive activation of sensory areas and slightly more extensive convergence in frontal and less in parietal regions. However, two separate statistical analyses using different convergence measures found no significant differences in print-speech convergence between the intermediate and advanced L2-learner groups in either language (or between languages in either group), indicating that print-speech convergence in L2 is unaffected by overall L2 proficiency level in skilled readers.

In monolinguals, print-speech convergence has been found to be a reliable and universal indicator of reading-related skills, invariant across age and languages (Shankweiler et al. 2008; Rueckl et al. 2015; Preston et al. 2016). In late bilingual-biliterates, participants displayed significantly more extensive print-speech convergence in frontal regions and less in parietal regions in L2 compared to L1, a finding considered to indicate more effortful reading 
in a new writing system (Brice et al. 2019). In these previous studies, participants had learnt to speak before they learnt to read in the same language, and their reading circuits were integrated with previously-established spoken language networks commensurate with their reading proficiency. In the current study, participants were skilled L1 readers learning an L2 with the same writing system as their L1 - reflecting the experience of many late bilinguals — and displayed no significant effect of L2 proficiency on print-speech convergence in either language. Thus we find that print-speech convergence is unaffected by overall language proficiency in skilled readers, at least when both languages have transparent orthographies.

\subsection{L1-L2 similarity decreased with greater L2 proficiency}

The similarity of L1 and L2 activation in classical language areas was significantly higher in the intermediate L2-learners compared to the advanced group. To test the hypothesis that L1-L2 similarity varies as a function of L2 proficiency, decreasing with increased L2 proficiency, we calculated within-group correlations between L1 and L2 laterality indices, and found high correlations in the intermediate group, which were not present in the advanced group. Finally, in L2 reading, we found large between-group differences in both dorsal and ventral pathways, while in L2 speech comprehension, there was a medium effect of proficiency in the ventral pathway and none in the dorsal.

Psycholinguistic theory has suggested that since late bilinguals acquire their L2 with reference to their L1, early stages of L2-learning entail dependency on the L1, which diminishes in later stages (Kroll and Stewart 1994; Kroll et al. 2010). Consistent with this idea, a recent word-learning study found that lexical items in an artificial language showed high neural pattern similarity with the native language (Li et al. 2018). In the current study, examining later stages in the language learning process, we found a strong correlation between L1 and L2 language networks in the intermediate language learners but a 
dissociation in the advanced learners. Thus, we infer that, though L1 and L2 share common neural bases, L2 proficiency modulates the similarity of their activation patterns in language learners.

Finally, we found a differential effect of L2-proficiency on the dorsal and ventral pathways across modalities. In both reading and speech comprehension, the dorsal pathway (IFG pars opercularis, STG, and IPL) is involved in phonological processing, i.e. grapheme to phoneme conversion in reading, and sound to articulatory-based representation in speech comprehension, while the ventral pathway (IFG pars orbitalis, IFG pars triangularis, vOTC) is involved in mapping of either written or spoken stimuli to its meaning. The more consistent effect of L2-proficiency on the ventral compared to the dorsal pathway in both reading and speech comprehension could be attributed to the fact that our participants' L1 and L2 have similar phonology but different morphology. Thus, given the prominent role of the ventral pathway in semantic processing, it is reasonable that we found a substantial effect of L2-proficiency on L2 activation of the ventral pathway regions in both reading and speech comprehension.

\subsection{Functional connectivity with control areas increased with greater L2 proficiency and exposure}

In our final analysis, we examined the recruitment of extra-linguistic areas such as the dlPFC and ACC in L2 comprehension. Pairwise functional connectivity analyses between language network and language control regions revealed no differences between the groups in L1 or in L2 speech comprehension, but showed differential functional coupling of the dlPFC with language regions during L2 reading. We found that advanced L2-learners exhibited significantly stronger coupling than did the intermediate L2-learners, indicating that 
coordination between the left dlPFC and language-related regions was significantly higher in L2 reading.

Language control is a crucial part of the language learning process, involving the recruitment of extra resources when comprehension and retrieval are effortful. Previous studies have found that lower L2 exposure between similarly proficient bilinguals was associated with more extensive prefrontal activation, particularly in the left hemisphere (Abutalebi et al. 2001; Perani et al. 2003; Indefrey 2006). We hypothesised that a diffuse activation of prefrontal areas in lower proficiency/exposure bilinguals may go hand in hand with weaker functional connectivity between the language regions and language control regions, and that functional connectivity would increase with greater proficiency and exposure. In the current study, we indeed found that the advanced L2-learner group with higher proficiency and exposure displayed greater coupling with the dlPFC than did intermediate learners. Thus we see that language learners who have achieved a high level of L2-proficiency — but still find comprehension more effortful than in their L1 (as measured by reaction times) — exhibit higher connectivity with the left dlPFC, a region implicated in effort and conflict resolution (Mansouri et al. 2009), than do language learners with lower L2 proficiency.

\subsection{Further questions}

In the second and third analyses - L1-L2 similarity and functional connectivity we found significant neural differences between groups in L2 reading, but smaller or insignificant differences in L2 speech comprehension - an effect not explained by inscanner task performance. Since the languages in the current study (Spanish and Basque) have considerably overlapping orthographic and phonological systems, the greater difference between groups in reading than in speech comprehension was unexpected and invites future 
scrutiny. Late language learners typically find reading easier than speech comprehension (Lund 1991; Mecartty 2000; Graham 2006), so if anything, we could have expected greater functional changes in the more demanding sub-skill, i.e. L2 speech comprehension. Since this is not the case, greater neural plasticity in L2 reading may be either the cause or the consequence of reading being experienced to be easier than speech comprehension. Future studies using longitudinal designs may be able to shed further light on this question.

Finally, are these differences between intermediate and advanced language learners indicative of functional reorganisation of L2 in even more proficient language learners? Our data indicated that similarity between L1 and L2 was less pronounced, while functional connectivity with control regions was stronger in advanced learners compared to intermediate L2-learners. Evidence from studies with different paradigms and analyses suggests that L1 and L2 access a common semantic hub and may thus converge completely, affording equivalent access in proficient bilinguals (Chee et al. 1999; Perani and Abutalebi 2005; Buchweitz et al. 2012; Correia et al. 2014), and that the need for recruitment of control regions diminishes in highly proficient bilinguals (Abutalebi and Green 2007). Though the evidence is limited and not directly comparable, it gives rise to the idea of a non-linear course of functional similarity between L1 and L2 with increasing L2 proficiency (Figure 5). Studies of structural learning-dependent plasticity in human adults and cellular and functional plasticity in animal models of learning have found a similar non-linear trajectory of expansion and re-normalisation (see Wenger et al. 2017 for review) with increasing task proficiency. Future studies designed to test this hypothesis in functional changes may well find an interesting parallel between functional plasticity and other levels of brain architecture and functioning. 


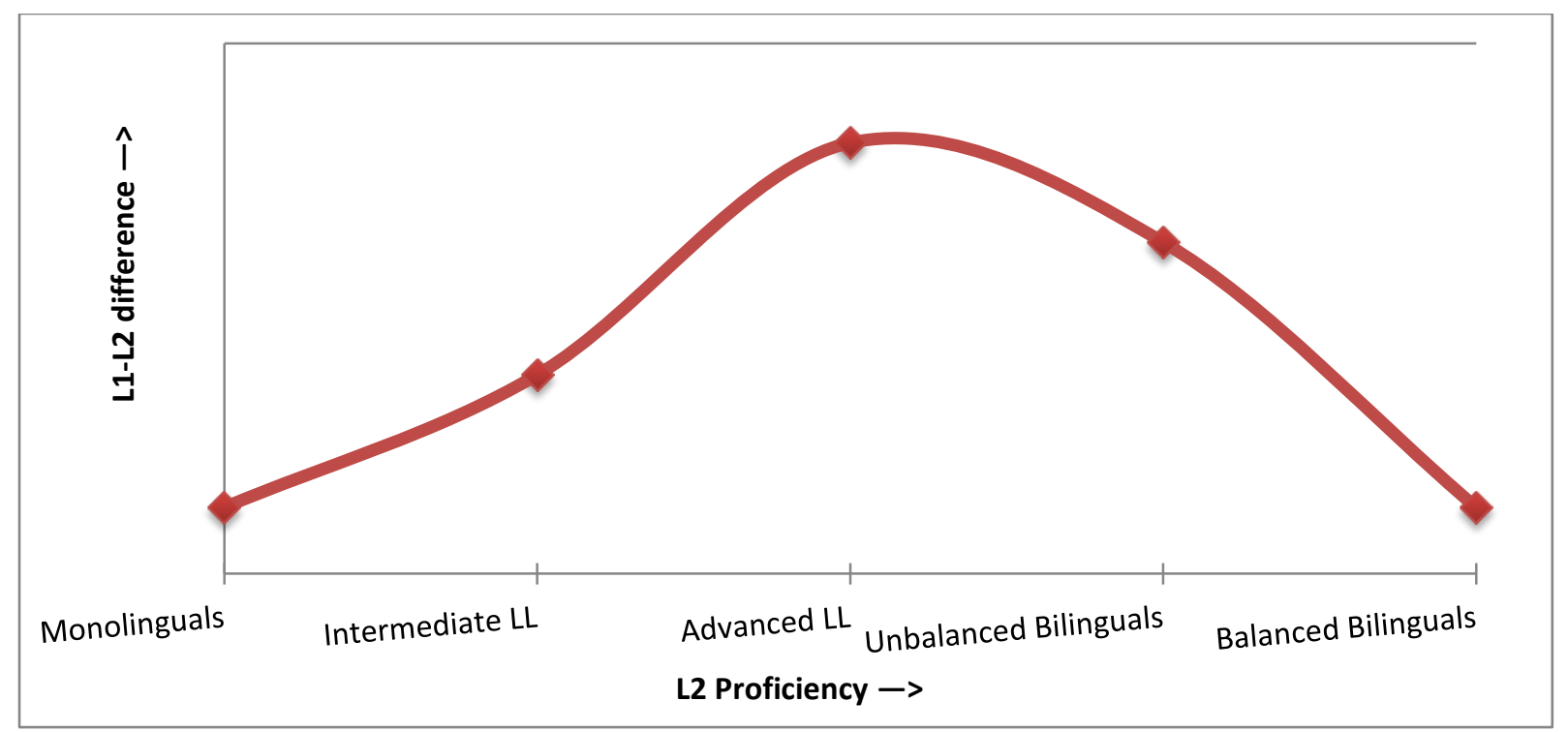

Figure 5. L1-L2 differences first increase and then decrease with increasing L2 proficiency $\mathrm{LL}=$ language learners

\subsection{Limitations}

One of the main limitations of the study is the low sample size. To address this issue, and in line with best statistical practices, we restricted the number of comparisons and used non-parametric statistics, within-subject measures, and effect sizes where appropriate. Second, though the cross-sectional design of the study was essential to capturing sufficient proficiency differences in a skill as complex as language-learning, this precluded more causal inferences or exploration of individual differences in plasticity. Finally, although the SpanishBasque combination of languages allowed us to control particularly well for extra-linguistic factors and linguistic factors besides morphology and syntax, it does not reflect the realities of most multilingual populations, making it tricky to compare studies of more contrasting languages such as English-Chinese or to directly generalise the results to other language experiences. 


\section{CONCLUSION}

While neural changes in young adults have consistently been associated with learning a completely new skill (see May 2011 for a review), lack of practice has been seen to reduce or even reverse some of these changes when the skill is not maintained, particularly in older adults (Boyke et al. 2008). The effects of ongoing practice of skills acquired in adulthood have received relatively little attention, and in the current study, we investigated neural changes concomitant with ongoing improvement of a complex skill such as language learning. By comparing intermediate and advanced adult language learners, we found that: print-speech convergence was unaffected by L2-proficiency differences, lower similarity between L1 and L2 activation was associated with higher L2 proficiency, and stronger functional connectivity with dIPFC during reading was seen with greater L2 proficiency and exposure, indicating that language learners well into adulthood display functional plasticity of language comprehension networks.

\section{ACKNOWLEDGEMENTS}

Kshipra Gurunandan received support from "la Caixa" Foundation (ID 100010434) through the fellowship LCF/BQ/DI17/11620005, and the European Union's Horizon 2020 research and innovation programme under the Marie Skłodowska-Curie grant agreement no. 713673. Manuel Carreiras was supported by project APCIN-2015-061-MultiLateral funded by the Spanish Ministry of Economy and Competitiveness (MINECO) [FLAG-ERA JTC 2015]. Pedro M. Paz-Alonso was supported by grants from MINECO [RYC-2014-15440, PGC2018-093408-B-I00], the Adinberri programme from the Diputación Foral de Gipuzkoa [OF301/2018], and the Neuroscience Research Projects programme from the Fundación Tatiana Pérez de Gúzman el Bueno. The research was also supported by funding from the 
Basque Government [BERC 2018-2021] and the Spanish State Research Agency through BCBL Severo Ochoa excellence accreditation [SEV-2015-0490]. The authors would like to thank Magda Altman for proofreading and helpful comments on the manuscript, and the reviewers for their insightful and constructive critique.

\section{REFERENCES}

[1] Abutalebi, J. and Green, D., 2007. Bilingual language production: The neurocognition of language representation and control. Journal of Neurolinguistics, 20(3), pp.242-275.

[2] Abutalebi, J., Annoni, J.M., Zimine, I., Pegna, A.J., Seghier, M.L., Lee-Jahnke, H., Lazeyras, F., Cappa, S.F. and Khateb, A., 2007. Language control and lexical competition in bilinguals: an event-related fMRI study. Cerebral Cortex, 18(7), pp.1496-1505.

[3] Abutalebi, J., Cappa, S.F. and Perani, D., 2001. The bilingual brain as revealed by functional neuroimaging. Bilingualism: Language and Cognition, 4(2), pp.179-190.

[4] Barbeau, E.B., Chai, X.J., Chen, J.K., Soles, J., Berken, J., Baum, S., Watkins, K.E., Klein, D., 2017. The role of the left inferior parietal lobule in second language learning: An intensive language training fMRI study. Neuropsychologia 98, 169-176.

[5] Boyke, J., Driemeyer, J., Gaser, C., Büchel, C. and May, A., 2008. Training-induced brain structure changes in the elderly. Journal of Neuroscience, 28(28), pp.7031-7035.

[6] Bradshaw, A.R., Bishop, D.V. and Woodhead, Z.V., 2017. Methodological considerations in assessment of language lateralisation with fMRI: a systematic review. PeerJ, 5, p.e3557.

[7] Brice, H., Mencl, W.E., Frost, S.J., Bick, A.S., Rueckl, J.G., Pugh, K.R., Frost, R., 2019. Neurobiological signatures of L2 proficiency: Evidence from a bi-directional crosslinguistic study. J. Neurolinguistics 50, 7-16. 
[8] Buchweitz, A., Shinkareva, S.V., Mason, R.A., Mitchell, T.M. and Just, M.A., 2012. Identifying bilingual semantic neural representations across languages. Brain and Language, 120(3), pp.282-289.

[9] Casanova, R., Srikanth, R., Baer, A., Laurienti, P.J., Burdette, J.H., Hayasaka, S., Flowers, L., Wood, F. and Maldjian, J.A., 2007. Biological parametric mapping: a statistical toolbox for multimodality brain image analysis. Neuroimage, 34(1), pp.137-143.

[10] Chai, X.J., Berken, J.A., Barbeau, E.B., Soles, J., Callahan, M., Chen, J.K., Klein, D., 2016. Intrinsic functional connectivity in the adult brain and success in second-language learning. J. Neurosci. 36 (3), 755-761.

[11] Chee, M.W., Hon, N., Lee, H.L. and Soon, C.S., 2001. Relative language proficiency modulates BOLD signal change when bilinguals perform semantic judgments. Neuroimage, 13(6), pp.1155-1163.

[12] Chee, M.W., Tan, E.W. and Thiel, T., 1999. Mandarin and English single word processing studied with functional magnetic resonance imaging. Journal of Neuroscience, 19(8), pp.3050-3056.

[13] Cocosco, C.A., Kollokian, V., Kwan, R.K.S., Pike, G.B. and Evans, A.C., 1997. Brainweb: Online interface to a 3D MRI simulated brain database. In NeuroImage.

[14] Correia, J., Formisano, E., Valente, G., Hausfeld, L., Jansma, B. and Bonte, M., 2014. Brain-based translation: fMRI decoding of spoken words in bilinguals reveals languageindependent semantic representations in anterior temporal lobe. Journal of Neuroscience, 34(1), pp.332-338.

[15] Dale, A.M., 1999. Optimal experimental design for event-related fMRI. Human Brain Mapping, 8(2-3), pp.109-114. 
[16] Dehaene, S., Dupoux, E., Mehler, J., Cohen, L., Paulesu, E., Perani, D., Van de Moortele, P.F., Lehéricy, S. and Le Bihan, D., 1997. Anatomical variability in the cortical representation of first and second language. Neuroreport, 8(17), pp.3809-3815.

[17] Fisher, R.A., 1921. On the probable error of a coefficient of correlation deduced from a small sample. Metron, 1, pp.3-32.

[18] Friederici, A.D., 2012. The cortical language circuit: from auditory perception to sentence comprehension. Trends in Cognitive Sciences, 16(5), pp.262-268.

[19] Frost, S.J., Sandak, R., Mencl, W.E., Landi, N., Rueckl, J.G., Katz, L. and Pugh, K.R., 2009. Mapping the word reading circuitry in skilled and disabled readers. How children learn to read: Current issues and new directions in the integration of cognition, neurobiology and genetics of reading and dyslexia research and practice, pp.3-19.

[20] Golestani, N. and Zatorre, R.J., 2004. Learning new sounds of speech: reallocation of neural substrates. Neuroimage, 21(2), pp.494-506.

[21] Golestani, N. and Zatorre, R.J., 2009. Individual differences in the acquisition of second language phonology. Brain and Language, 109(2-3), pp.55-67.

[22] Graham, S., 2006. Listening comprehension: The learners' perspective. System, 34(2), pp.165-182.

[23] Hagoort, P., 2013. MUC (memory, unification, control) and beyond. Frontiers in Psychology, 4, p.416.

[24] Hernandez, A.E., 2009. Language switching in the bilingual brain: What's next? Brain and Language, 109(2-3), pp.133-140.

[25] Hickok, G. and Poeppel, D., 2004. Dorsal and ventral streams: a framework for understanding aspects of the functional anatomy of language. Cognition, 92(1-2), pp.6799. 
[26] Hull, R. and Vaid, J., 2007. Bilingual language lateralization: A meta-analytic tale of two hemispheres. Neuropsychologia, 45(9), pp.1987-2008.

[27] Indefrey, P., 2006. A meta-analysis of hemodynamic studies on first and second language processing: Which suggested differences can we trust and what do they mean? Language Learning, 56, pp.279-304.

[28] Jobard, G., Crivello, F. and Tzourio-Mazoyer, N., 2003. Evaluation of the dual route theory of reading: a metanalysis of 35 neuroimaging studies. Neuroimage, 20(2), pp.693712.

[29] Kaplan, E.F., Goodglass, H. and Weintraub, S., 1983. The Boston naming test. 2nd. Philadelphia: Lea \& Febiger.

[30] Kim, K.H., Relkin, N.R., Lee, K.M. and Hirsch, J., 1997. Distinct cortical areas associated with native and second languages. Nature, 388(6638), p.171.

[31] Kroll, J.F. and Stewart, E., 1994. Category interference in translation and picture naming: Evidence for asymmetric connections between bilingual memory representations. Journal of Memory and Language, 33(2), pp.149-174.

[32] Kroll, J.F., Van Hell, J.G., Tokowicz, N. and Green, D.W., 2010. The Revised Hierarchical Model: A critical review and assessment. Bilingualism: Language and Cognition, 13(3), pp.373-381.

[33] Lau, E.F., Phillips, C. and Poeppel, D., 2008. A cortical network for semantics:(de) constructing the N400. Nature Reviews Neuroscience, 9(12), p.920.

[34] Li, H., Qu, J., Chen, C., Chen, Y., Xue, G., Zhang, L., Lu, C., Mei, L., 2018. Lexical learning in a new language leads to neural pattern similarity with word reading in native language. Hum. Brain Mapp. 40 (1), 98-109.

[35] Liu, H. and Cao, F., 2016. L1 and L2 processing in the bilingual brain: A metaanalysis of neuroimaging studies. Brain and Language, 159, pp.60-73. 
[36] López-Barroso, D., Catani, M., Ripollés, P., Dell'Acqua, F., Rodríguez-Fornells, A. and de Diego-Balaguer, R., 2013. Word learning is mediated by the left arcuate fasciculus. Proceedings of the National Academy of Sciences, 110(32), pp.13168-13173.

[37] Lund, R.J., 1991. A comparison of second language listening and reading comprehension. The Modern Language Journal, 75(2), pp.196-204.

[38] Mansouri, F.A., Tanaka, K. and Buckley, M.J., 2009. Conflict-induced behavioural adjustment: a clue to the executive functions of the prefrontal cortex. Nature Reviews Neuroscience, 10(2), p.141.

[39] Marian, V., Shildkrot, Y., Blumenfeld, H.K., Kaushanskaya, M., Faroqi-Shah, Y. and Hirsch, J., 2007. Cortical activation during word processing in late bilinguals: similarities and differences as revealed by functional magnetic resonance imaging. Journal of Clinical and Experimental Neuropsychology, 29(3), pp.247-265.

[40] Mårtensson, J., Eriksson, J., Bodammer, N.C., Lindgren, M., Johansson, M., Nyberg, L., Lövdén, M., 2012. Growth of language-related brain areas after foreign language learning. NeuroImage 63 (1), 240-244.

[41] May, A., 2011. Experience-dependent structural plasticity in the adult human brain. Trends in Cognitive Sciences, 15(10), pp.475-482.

[42] Mazaika, P.K., Hoeft, F., Glover, G.H. and Reiss, A.L., 2009. Methods and software for fMRI analysis of clinical subjects. Neuroimage, 47(Suppl 1), p.S58.

[43] Mecartty, F.H., 2000. Lexical and grammatical knowledge in reading and listening comprehension by foreign language learners of Spanish. Applied Language Learning, 11(2), pp.323-348.

[44] Mechelli, A., Crinion, J.T., Noppeney, U., O'doherty, J., Ashburner, J., Frackowiak, R.S. and Price, C.J., 2004. Neurolinguistics: structural plasticity in the bilingual brain. Nature, 431(7010), p.757. 
[45] Nichols, T.E. and Holmes, A.P., 2001. Nonparametric analysis of PET functional neuroimaging experiments: a primer. Human Brain Mapping, 15, pp.1-25.

[46] Oliver, M., Carreiras, M. and Paz-Alonso, P.M., 2016. Functional dynamics of dorsal and ventral reading networks in bilinguals. Cerebral Cortex, 27(12), pp.5431-5443.

[47] Penny, W.D., Friston, K.J., Ashburner, J.T., Kiebel, S.J. and Nichols, T.E. eds., 2011. Statistical parametric mapping: the analysis of functional brain images. Elsevier.

[48] Perani, D. and Abutalebi, J., 2005. The neural basis of first and second language processing. Current Opinion in Neurobiology, 15(2), pp.202-206.

[49] Perani, D., Abutalebi, J., Paulesu, E., Brambati, S., Scifo, P., Cappa, S.F. and Fazio, F., 2003. The role of age of acquisition and language usage in early, high-proficient bilinguals: An fMRI study during verbal fluency. Human Brain Mapping, 19(3), pp.170182.

[50] Perani, D., Paulesu, E., Galles, N.S., Dupoux, E., Dehaene, S., Bettinardi, V., Cappa, S.F., Fazio, F. and Mehler, J., 1998. The bilingual brain. Proficiency and age of acquisition of the second language. Brain: a journal of neurology, 121(10), pp.1841-1852.

[51] Plante, E., Almryde, K., Patterson, D.K., Vance, C.J. and Asbjørnsen, A.E., 2015. Language lateralization shifts with learning by adults. Laterality: Asymmetries of Body, Brain and Cognition, 20(3), pp.306-325.

[52] Preston, J.L., Molfese, P.J., Frost, S.J., Mencl, W.E., Fulbright, R.K., Hoeft, F., Landi, N., Shankweiler, D. and Pugh, K.R., 2016. Print-speech convergence predicts future reading outcomes in early readers. Psychological Science, 27(1), pp.75-84.

[53] Rissman, J., Gazzaley, A. and D'Esposito, M., 2004. Measuring functional connectivity during distinct stages of a cognitive task. Neuroimage, 23(2), pp.752-763.

[54] Rueckl, J.G., Paz-Alonso, P.M., Molfese, P.J., Kuo, W.J., Bick, A., Frost, S.J., Hancock, R., Wu, D.H., Mencl, W.E., Duñabeitia, J.A. and Lee, J.R., 2015. Universal 
brain signature of proficient reading: Evidence from four contrasting languages. Proceedings of the National Academy of Sciences, 112(50), pp.15510-15515.

[55] Schlaggar, B.L. and McCandliss, B.D., 2007. Development of neural systems for reading. Annu. Rev. Neurosci., 30, pp.475-503.

[56] Schlegel, A.A., Rudelson, J.J., Tse, P.U., 2012. White matter structure changes as adults learn a second language. J. Cogn. Neurosci. 24 (8), 1664-1670.

[57] Shankweiler, D., Mencl, W.E., Braze, D., Tabor, W., Pugh, K.R. and Fulbright, R.K., 2008. Reading differences and brain: Cortical integration of speech and print in sentence processing varies with reader skill. Developmental Neuropsychology, 33(6), pp.745-775.

[58] Singh, N.C., Cherodath, S., Sumathi, T.A., Kasera, R., Currawala, K., Kar, B. and Oberoi, G., 2016. Reading skills in children provided simultaneous instruction in two distinct writing systems. Multilingualism, Literacy and Dyslexia: Breaking down barriers for educators, p.200.

[59] Stein, M., Federspiel, A., Koenig, T., Wirth, M., Lehmann, C., Wiest, R., Strik, W., Brandeis, D., Dierks, T., 2009. Reduced frontal activation with increasing 2nd language proficiency. Neuropsychologia 47 (13), 2712-2720.

[60] Stein, M., Federspiel, A., Koenig, T., Wirth, M., Strik, W., Wiest, R., Brandeis, D., Dierks, T., 2012. Structural plasticity in the language system related to increased second language proficiency. Cortex 48 (4), 458-465.

[61] Talairach, J. and Tournoux, P., 1988. Co-planar stereotaxic atlas of the human brain: 3-dimensional proportional system: an approach to cerebral imaging.

[62] Tzourio-Mazoyer, N., Landeau, B., Papathanassiou, D., Crivello, F., Etard, O., Delcroix, N., Mazoyer, B. and Joliot, M., 2002. Automated anatomical labeling of activations in SPM using a macroscopic anatomical parcellation of the MNI MRI singlesubject brain. Neuroimage, 15(1), pp.273-289. 
[63] Wang, Y., Sereno, J.A., Jongman, A. and Hirsch, J., 2003. fMRI evidence for cortical modification during learning of Mandarin lexical tone. Journal of Cognitive Neuroscience, 15(7), pp.1019-1027.

[64] Wartenburger, I., Heekeren, H.R., Abutalebi, J., Cappa, S.F., Villringer, A. and Perani, D., 2003. Early setting of grammatical processing in the bilingual brain. Neuron, 37(1), pp.159-170.

[65] Wenger, E., Brozzoli, C., Lindenberger, U. and Lövdén, M., 2017. Expansion and renormalization of human brain structure during skill acquisition. Trends in Cognitive Sciences, 21(12), pp.930-939.

[66] Wilke, M. and Lidzba, K., 2007. LI-tool: a new toolbox to assess lateralization in functional MR-data. Journal of Neuroscience Methods, 163(1), pp.128-136.

[67] Wong, P.C., Perrachione, T.K. and Parrish, T.B., 2007. Neural characteristics of successful and less successful speech and word learning in adults. Human Brain Mapping, 28(10), pp.995-1006.

[68] Xiang, H., van Leeuwen, T.M., Dediu, D., Roberts, L., Norris, D.G., Hagoort, P., 2015. L2-proficiencydependent laterality shift in structural connectivity of brain language pathways. Brain Connect. 5 (6), 349-361. 